\title{
Clinical Impact of Rest Dual-energy Computed Tomography Myocardial Perfusion in Patients with Coronary Artery Disease
}

\author{
STEFAN BAUMANN ${ }^{1}$, MARLON RUTSCH ${ }^{1}$, TOBIAS BECHER ${ }^{1}$, PHILIPP KRYEZIU ${ }^{1}$, \\ HOLGER HAUBENREISSER $^{2}$, NILS VOGLER ${ }^{2}$, CELI ANNE SCHOENIKE ${ }^{3}$, MARTIN BORGGREFE ${ }^{1}$, \\ STEFAN O. SCHOENBERG ${ }^{2}$, IBRAHIM AKIN ${ }^{1}$, THOMAS HENZLER ${ }^{2}$ and DIRK LOSSNITZER ${ }^{1}$ \\ ${ }^{1}$ First Department of Medicine-Cardiology, University Medical Centre Mannheim, Mannheim, \\ Germany and DZHK (German Centre for Cardiovascular Research), \\ partner site Heidelberg/Mannheim, Mannheim, Germany; \\ ${ }^{2}$ Institute of Clinical Radiology and Nuclear Medicine, \\ University Medical Centre Mannheim, Mannheim, Germany; \\ ${ }^{3}$ Medical Faculty Mannheim, University of Heidelberg, Mannheim, Germany
}

\begin{abstract}
Background/Aim: To evaluate the hypothesis that patients with suspected coronary artery disease (CAD) assessed using rest dual-energy computed tomography-derived myocardial perfusion imaging (DECT-P), could have fewer invasive coronary angiographies (ICA), showing nonobstructive CAD. Materials and Methods: Patients who had undergone coronary computed tomography angiography (cCTA), rest DECT-P and ICA were analyzed. Results: We evaluated 51 patients $(62.7 \%$ males, mean age $51.6 \pm 12.8$ years). Rest DECT-P identified perfusion defects in three (10.7\%) of the 28 patients with cCTA negative for luminal stenosis and in $10(43.5 \%)$ of the 23 patients with cCTA positive for luminal stenosis. In total, 21 patients underwent both cCTA and ICA, of which seven (33.3\%) showed obstructive CAD. Rest DECT-P revealed false-negative results in four cases (19.1\%) and false-positive results in six cases (28.6\%). Conclusion: Adding rest DECT-P to cCTA has no incremental diagnostic value over cCTA alone, to exclude haemodynamically significant CAD. Therefore, a rest-stress-DECT-P protocol or a CT-based FFR calculation might be a promising concept to improve diagnostic accuracy in a real clinical setting.
\end{abstract}

This article is freely accessible online.

Correspondence to: Dr. Thomas Henzler, Institute of Clinical Radiology and Nuclear Medicine, University Medical Center Mannheim, Medical Faculty Mannheim, Heidelberg University, Theodor-Kutzer-Ufer 1-3, 68167 Mannheim, Germany. Tel: +49 6213832067, Fax: +496213831910,e-mail: thomas.henzler@ medma.uni-heidelberg.de

Key Words: Coronary CT angiography, coronary artery disease, myocardial perfusion imaging, dual-energy CT, myocardial ischemia.
Current guidelines in the management of stable coronary artery disease $(\mathrm{CAD})$ recommend a non-invasive functional test before considering invasive coronary angiography (ICA) for patients with intermediate pre-test probability (1). Due to the high sensitivity and negative predictive value, coronary computed tomography angiography (cCTA) is a clinically-established noninvasive diagnostic imaging modality to rule-out CAD. However, the specificity and positive predictive value of cCTA is still limited due to the poor correlation of cCTA-detected coronary artery stenosis and myocardial ischaemia.

One avenue for assessment of the myocardial blood supply consists of dual-energy computed tomography (DECT) acquisitions during first-pass arterial enhancement. Dualenergy computed tomography-derived myocardial perfusion imaging (DECT-P) is a "one-stop shop" approach closing the gap between anatomical and functional aspects within a single imaging modality and could reduce false-positive results of cCTA (2). As rest DECT-P is derived from the same datasets as standard cCTA, it requires no additional contrast media, radiation exposure, or stress agents, which represents a substantial advantage, when compared to stress protocols. Studies have compared DECT-P with single-photon emission computed tomography (SPECT), magnetic resonance imaging (MRI), and ICA in rest or rest-stress acquisition with first or second generation dual-source scanners and found an incremental value over cCTA alone (3-6). However, the data for rest-only acquisition in DECT-P is rather sparse and needs further investigation regarding the clinical utility and its effect on patient care in a real clinical setting.

Thus, the purpose of the present study was to test the hypothesis that patients with suspected CAD evaluated using a rest DECT-P strategy would potentially have fewer coronary angiographies without obstructive CAD than patients who were evaluated based on standard cCTA protocols. 


\section{Materials and Methods}

Patient population, study design and analysis. The Health Insurance Portability and Accountability Act (HIPAA) compliant studyprotocol is in accordance with the Declaration of Helsinki and was approved by our local ethics committee. Written informed consent was obtained from all patients. From February 2016 until October 2016 we enrolled 51 patients with suspected CAD and with low to intermediate pre-test probability for CAD in our prospective singlecentre study. We selected patients in accordance with the 2013 guidelines of the European Society of Cardiology (ESC) on the management of stable CAD.

Exclusion criteria were: Renal insufficiency, contrast agent allergy, ST-segment elevation or recent left bundle branch block detected in electrocardiography (ECG), known myocardial infarction, percutaneous coronary intervention (PCI) or bypass operation within the past six months, or a significant increase of high-sensitivity-troponin I (>30\%). Participants were referred for a standard cCTA in combination with a rest DECT-P for myocardial perfusion assessment. If cCTA showed stenosis that might be of haemodynamic significance or of unclear origin, participants subsequently proceeded to ICA. Finally, in order to test the study, the results of patients with non-obstructive findings in ICA were compared to the results of the same patients' DECT-P findings.

Computed tomography $(C T)$ acquisition protocol. All CT scans were performed using a 192-slice, third-generation, dual-source CT system (Siemens Somatom Force, Forchheim, Germany). The protocol consisted of a non-enhanced, single-energy standard scan for calcium scoring, subsequently followed by a cCTA with dualenergy acquisition (Figure 1A). Acquisition parameters for the nonenhanced scan were: tube voltage $120 \mathrm{kV}$; detector collimation $2 \times 192 \times 0.6 \mathrm{~mm}$, as well as the use of prospective ECG-triggering. The reconstruction parameters used for calcium scoring were: slice thickness $3.0 \mathrm{~mm}$, increment $1.5 \mathrm{~mm}$ and Qr36 iterative reconstruction kernel. Acquisition parameters for the dual-energy cCTA were: tube voltage $90 \mathrm{kV}$ (tube A), tube voltage $150 \mathrm{kV}$ with additional tin filter (tube B) and, for both tube $\mathrm{A}$ and tube $\mathrm{B}$, an automatic tube current modulation; detector collimation $2 \times 192 \times 0.6$ $\mathrm{mm}$. The reconstruction parameters were: slice thickness $0.6 \mathrm{~mm}$, increment $0.5 \mathrm{~mm}$ and Bv40 kernel. All cCTA acquisitions were performed with retrospective ECG-gating and bolus tracking with the trigger region-of-interest (ROI) placed in the descending aorta with a 100 Hounsfield Unit (HU) threshold. No ECG-dependent tube current modulation was applied. Eighty millilitres of intravenous contrast (Imeron ${ }^{\circledR}$ 400, Bracco, Milan, Italy) were administered through an $18 \mathrm{G}$ cubital catheter, which was followed by a $50 \mathrm{ml}$ saline flush. Both were injected at a rate of five $\mathrm{ml} / \mathrm{sec}$. Control of cardiac contraction frequency was achieved by intravenous administration of beta-blockers, targeting a heart rate of $\leq 65$ beats $/ \mathrm{min}$. Sublingual nitrates were administered to ensure coronary vasodilatation. First-pass DECT-P was reconstructed from the raw data of the cCTA acquisition (Figure 1B).

CT Image analysis. All examinations were evaluated by physicians with expertise in cardiovascular imaging. Evaluation of cCTA was performed using axial images as well as multi-planar and curved reformations. Degree of stenosis in cCTA was quantified according to the Society of Cardiovascular Computed Tomography (SCCT) guidelines, with significant luminal narrowing defined as $\geq 70 \%$ (7).
For evaluation of first-pass DECT-P data, iodine maps were calculated using a dedicated post-processing work station (Syngo.Via VB10B, Siemens Healthcare, Forchheim, Germany). Iodine attenuation was normalized to the left ventricle. Myocardial perfusion defects were defined as circumscribed areas of decreased or absent iodine content within the left ventricular wall, relative to the remainder of the myocardium. Assignment of the affected segment was based on the American Heart Association's 17-segment model of visual assessment of myocardial perfusion defects shown in the diastolic phase.

ICA with measurement of fractional flow reserve (FFR). ICA was performed by an experienced senior interventional cardiologist according to standard procedures (Figure 1C). FFR measurement (PressureWire Certus, St. Jude Medical; St. Paul, Minnesota, USA) was performed during ICA when the haemodynamic significance of a coronary lesion remained visually unclear. A diagnosis of ischaemia could be declared when the FFR was $\leq 0.80$.

\section{Results}

We evaluated 51 patients (62.7\% males, $51.6 \pm 12.8$ years) with a mean Agatston score of $145.7 \pm 507.6$ and a mean updated Diamond-Forrester score of $26.8 \pm 13.1 \%$. The amount of administered CT contrast medium was $84.9 \pm 13.8$ $\mathrm{ml}$ and the dose-length product for cCTA was $642.5 \pm 154.4$ $\mathrm{mGy}^{*} \mathrm{~cm}$ for cCTA and DECT-P. Further baseline characteristics are shown in Table I.

cCTA identified three $(5.9 \%)$ patients with severe coronary stenosis $(\geq 70 \%)$, nine $(17.6 \%)$ with moderate stenosis $(50-69 \%)$, six $(11.8 \%)$ with mild stenosis $(25-49 \%)$, five $(9.8 \%)$ with minimal stenosis $(<25 \%)$ and $28(54.9 \%)$ without luminal stenosis.

Rest DECT-P identified perfusion defects in three (10.7\%) of the 28 patients with cCTA negative for luminal stenosis, and in ten $(43.5 \%)$ of the 23 patients with cCTA positive for luminal stenosis.

In total, 21 patients underwent both cCTA and ICA, seven (33.3\%) of whom showed obstructive CAD in ICA defined as visually high-grade stenosis $(>70 \%)$ or invasive FFR $\leq 0.80$. Rest DECT-P revealed false negative results in four cases $(19.1 \%)$ and false positive results in six cases $(28.6 \%)$, regarding ICA results as a reference (Figure 2).

\section{Discussion}

Unfortunately, our study results were underwhelming, and demonstrated that adding rest DECT-P to cCTA has no incremental diagnostic value over cCTA alone to exclude haemodynamically significant CAD in patients with low to intermediate pre-test probability for CAD.

While cCTA is an excellent diagnostic method to rule out $\mathrm{CAD}$, its specificity remains relatively low and is unable to spare patients without obstructive CAD unnecessary ICA. In the multi-centre PLATFORM trial, non-invasive functional 

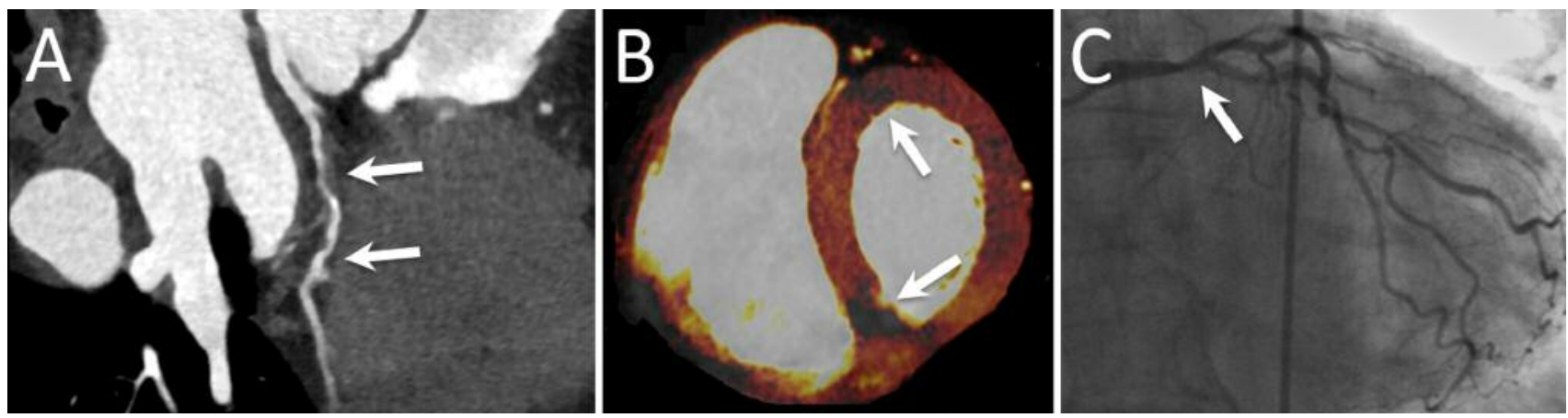

Figure 1. Case of a 59-year-old female patient with atypical chest pain, and a pretest probability of 20\%. (A) cCTA show several moderate stenoses of the LAD (arrows). (B) DECT-P show minor iodine distribution within basal LAD and RCA territory as a sign of haemodynamic significance (arrows). (C) ICA show severe artery disease of all three vessels. Subtotal stenosis of RCA, significant stenosis of the left main trunk (arrow) and 75\% stenosis of mid-RCX and ramus marginalis. cCTA: Coronary computed tomography; DECT-P: dual-energy computed tomography-derived myocardial perfusion imaging; LAD: left anterior descending; RCA: right coronary artery; RCX: Ramus circumflexus; ICA: invasive coronary angiography.

\section{Results of DECT-P perfusion in non- obstructive ICA findings}

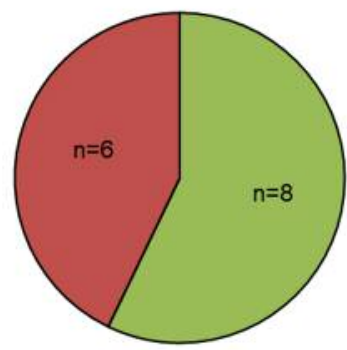

\section{Results of DECT-P perfusion in obstructive ICA findings}

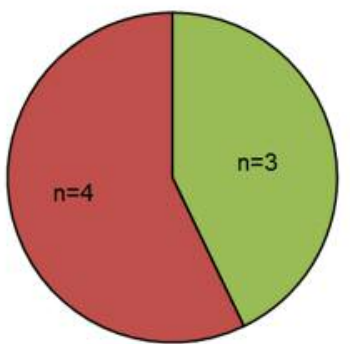

口DECT-P correct

口DECT-P incorrect

Figure 2. Results of DECT-P compared to ICA findings. Rest DECT-P revealed false-negative results in four cases (19.1\%) and false-positive results in six cases (28.6\%), compared to ICA results, as a reference. DECT-P: Dual-energy computed tomography-derived myocardial perfusion imaging; ICA: invasive coronary angiography.

testing was feasible and a safe alternative to ICA, and was associated with a significantly lower rate of ICA showing no obstructive CAD. Of the 187 PLATFORM patients who underwent ICA, 137 (73.3\%) did not show obstructive CAD (8). Following our initial hypothesis, we hypothesized that ICA examination could have potentially been avoided, according to results of the rest DECT-P assessment, in comparison to an evaluation strategy based on standard cCTA.

However, the chosen strategy led to disappointing results, as the rest DECT showed six false-positive results and, even more importantly, four false-negative results, which could lead to serious consequences for patient safety and prognosis. A dual-energy, static first-pass imaging approach makes the mapping of iodine distribution possible, and serves as a surrogate of myocardial perfusion. Additionally, it makes the quantification of myocardial blood supply possible. Like other functional imaging methods, a standard dual-energy cardiac examination is a combination of rest, stress and late enhancement (9). However, the major limitation of this conventional myocardial perfusion remains to be its additional radiation dose, as well as the required use of stress agents. This is associated with greater effort on the part of the medical professional, due to longer duration of examination and monitoring of the patient, and, therefore, is not always feasible in a real clinical setting. For this reason, we decided to use first-pass arterial rest dual-energy CT, which, compared to stress acquisition, does not require further radiation dose, contrast agent or medication, and, 
Table I. Baseline characteristics and findings of coronary computed tomography, myocardial perfusion imaging and invasive coronary angiography.

\begin{tabular}{|c|c|}
\hline \multicolumn{2}{|l|}{ Baseline characteristics } \\
\hline Age, years & $51.6 \pm 12.8$ \\
\hline Male & $32(62.7 \%)$ \\
\hline Body mass index $\left(\mathrm{kg} / \mathrm{m}^{2}\right)$ & $31.0 \pm 6.7$ \\
\hline \multicolumn{2}{|l|}{ Cardiovascular Risk Factors } \\
\hline Hypertension & $30(58.8 \%)$ \\
\hline Smoking & $28(54.9 \%)$ \\
\hline Family history of CAD & $20(39.2 \%)$ \\
\hline Diabetes & $12(23.5 \%)$ \\
\hline Hyperlipidaemia & $8(15.7 \%)$ \\
\hline Updated Diamond-Forrester risk score ${ }^{1}$ & $22.1 \pm 16.8$ \\
\hline \multicolumn{2}{|l|}{ Lipid Profile } \\
\hline Low-density lipoprotein, mg/dl & $128.0(111.0-149.0)$ \\
\hline High-density lipoprotein, mg/dl & $40.0(35.3-45.8)$ \\
\hline Triglycerides, mg/dl & $163.5(121.3-231.0)$ \\
\hline \multicolumn{2}{|l|}{ Coronary Computed Tomography } \\
\hline \multicolumn{2}{|l|}{ Data of cCTA Acquisition } \\
\hline Dose length product, $\mathrm{mGy}{ }^{*} \mathrm{~cm}$ & $642.5 \pm 154.4$ \\
\hline CTDIvol, mGy & $43.2 \pm 9.7$ \\
\hline Agatston score & $145.7 \pm 507.6$ \\
\hline \multicolumn{2}{|l|}{ Stenosis Grade in cCTA ${ }^{2}$} \\
\hline Normal (absence of plaque and no stenosis) & $28(54.9 \%)$ \\
\hline Minimal (plaque with $<25 \%$ stenosis) & $5(9.8 \%)$ \\
\hline Mild $(25 \%$ to $49 \%)$ & $6(11.8 \%)$ \\
\hline Moderate $(50 \%$ to $69 \%)$ & $9(17.6 \%)$ \\
\hline Severe $(70 \%$ to $99 \%)$ & $3(5.9 \%)$ \\
\hline \multicolumn{2}{|l|}{ Target Vessel, $\mathrm{n}=33$} \\
\hline Left main trunk, LMT & $1(3.0 \%)$ \\
\hline Left anterior descending, LAD & $21(63.6 \%)$ \\
\hline Ramus circumflexus, RCX & $7(21.2 \%)$ \\
\hline Right coronary artery, RCA & $4(12.1 \%)$ \\
\hline \multicolumn{2}{|l|}{ Myocardial Perfusion Imaging (DECT-P) } \\
\hline No perfusion defect & $38(74.5 \%)$ \\
\hline Perfusion defect & $13(25.5 \%)$ \\
\hline \multicolumn{2}{|l|}{ Invasive Coronary Angiography, $n=20$} \\
\hline No $\mathrm{CAD}^{3}$ & $5(25.0 \%)$ \\
\hline 1 vessel disease ${ }^{4}$ & $4(20.0 \%)$ \\
\hline 2 vessel disease & $4(20.0 \%)$ \\
\hline 3 vessel disease & $7(35.0 \%)$ \\
\hline \multicolumn{2}{|l|}{ Findings of FFR Measurement, $n=13$} \\
\hline Ischaemic lesions with FFR $\leq 0.80$ & $4(30.8 \%)$ \\
\hline Non-ischaemic lesions with FFR $>0.80$ & $9(69.2 \%)$ \\
\hline
\end{tabular}

Unless otherwise specified, data are numbers of patients, with percentages in parentheses. Data are mean \pm standard deviation (SD) or median with interquartile ranges. ${ }^{1}$ according to Genders et al. (13), ${ }^{2}$ according to SCCT guidelines (7), ${ }^{3}$ defined as coronary lesions $<50 \%$ stenosis, ${ }^{4}$ defined as coronary lesions $>50 \%$ stenosis.

thus, is a promising approach. Meinel et al. compared the relative contributions of rest, stress and delayed acquisitions, with the accuracy of DECT-P and demonstrated an impressively high specificity $(98.0 \%)$ and positive predictive value (94.0\%) for rest-only DECT-P (6). Furthermore, they postulate a dose-reduction of $68.0 \%$ in a rest approach, compared to comprehensive protocol of rest, stress and late enhancement. Comparison of radiation between static and dynamic perfusion shows higher doses for dynamic protocols $(10.1 \pm 15.5 \mathrm{mSv}, 11.5 \pm 3.5 \mathrm{mSV})$. A direct comparison of the mean radiation dose in our study of $642.5 \pm 154.4 \mathrm{mGy}^{*} \mathrm{~cm}$ to $1399.4 \pm 314.2 \mathrm{mGy} * \mathrm{~cm}$ in a dynamic rest-only approach (10) clearly demonstrates the advantages of static perfusion, with regard to radiation exposure. A further radiation sparing aspect in our study is the use of a recent third-generation dual-source scanner. Dosimetric parameters for cCTA and coronary calcium scoring is lower in third-generation scanners $(31.5 \pm 8.7 \mathrm{mGy} * \mathrm{~cm}, 22.7 \pm 4.9 \mathrm{mGy} * \mathrm{~cm})$ than in second-generation scanners $(65.4 \pm 21.2 \mathrm{mGy} * \mathrm{~cm}, 31.3 \pm 8.0$ $\mathrm{mGy} * \mathrm{~cm})(11)$.

Osawa et al. provided important contributions to the diagnostic value of DECT myocardial rest perfusion imaging (10). Furthermore, mixed assessment of HU values to detect abnormalities in perfusion is subjected to high tissue variability of HU values, because of deposition of fat or iron in the myocardium. Further studies are required to quantify perfusion defects in dual-energy CT imaging.

There were limitations in our study. First, the study population consists of a small, single-centre cohort, but is comparable with other studies in this research field. Furthermore, enrolment was stopped after non-promising interim analysis. Second, the percentage of haemodynamically-relevant coronary lesions is rather low, which might affect the level of significance. Third, ethically, it was not approved to perform ICA in patients with negative findings in cCTA/DECT-P to avoid complication in the ICA, nor was it approved to perform subsequent cCTA / DECT-P in patients with positive findings in ICA only for research purposes without clinical reason, due to the application of unjustified radiation exposure.

\section{Conclusion}

Our study demonstrated that adding rest DECT-P to cCTA did not fulfil our primary hypothesis for an incremental diagnostic value over cCTA alone. Thus, a rest-stress-DECT-P protocol, or a CT-based FFR calculation seems to be a promising concept to improve diagnostic accuracy in a real clinical setting (12).

\section{Conflicts of Interest}

All Authors declare that there are no conflicts of interest.

\section{Acknowledgements}

Supported by the DZHK (Deutsches Zentrum für Herz-KreislaufForschung - German Centre for Cardiovascular Research) and by the BMBF (Bildungsministerium für Bildung und Forschung German Ministry of Education and Research). 


\section{References}

1 Task Force M, Montalescot G, Sechtem U, Achenbach S, Andreotti F, Arden C, Budaj A, Bugiardini R, Crea F, Cuisset T, Di Mario C, Ferreira JR, Gersh BJ, Gitt AK, Hulot JS, Marx N, Opie LH, Pfisterer M, Prescott E, Ruschitzka F, Sabate M, Senior R, Taggart DP, van der Wall EE, Vrints CJ, Guidelines ESCCfP, Zamorano JL, Achenbach S, Baumgartner H, Bax JJ, Bueno H, Dean V, Deaton C, Erol C, Fagard R, Ferrari R, Hasdai D, Hoes AW, Kirchhof P, Knuuti J, Kolh P, Lancellotti P, Linhart A, Nihoyannopoulos P, Piepoli MF, Ponikowski P, Sirnes PA, Tamargo JL, Tendera M, Torbicki A, Wijns W, Windecker S, Document R, Knuuti J, Valgimigli M, Bueno H, Claeys MJ, Donner-Banzhoff N, Erol C, Frank H, FunckBrentano C, Gaemperli O, Gonzalez-Juanatey JR, Hamilos M, Hasdai D, Husted S, James SK, Kervinen K, Kolh P, Kristensen SD, Lancellotti P, Maggioni AP, Piepoli MF, Pries AR, Romeo F, Ryden L, Simoons ML, Sirnes PA, Steg PG, Timmis A, Wijns W, Windecker S, Yildirir A and Zamorano JL: 2013 esc guidelines on the management of stable coronary artery disease: The task force on the management of stable coronary artery disease of the european society of cardiology. Eur Heart $\mathbf{J}$ 34(38): 2949-3003, 2013.

2 Pelgrim GJ, Dorrius M, Xie X, den Dekker MA, Schoepf UJ, Henzler T, Oudkerk M and Vliegenthart R: The dream of a onestop-shop: Meta-analysis on myocardial perfusion ct. Eur J Radiol 84(12): 2411-2420, 2015.

3 Ko SM, Choi JW, Hwang HK, Song MG, Shin JK and Chee HK: Diagnostic performance of combined noninvasive anatomic and functional assessment with dual-source ct and adenosine-induced stress dual-energy ct for detection of significant coronary stenosis. AJR Am J Roentgenol 198(3): 512-520, 2012.

4 Wang R, Yu W, Wang Y, He Y, Yang L, Bi T, Jiao J, Wang Q, Chi L, Yu Y and Zhang Z: Incremental value of dual-energy ct to coronary ct angiography for the detection of significant coronary stenosis: Comparison with quantitative coronary angiography and single photon emission computed tomography. Int J Cardiovasc Imaging 27(5): 647-656, 2011.

5 De Cecco CN, Harris BS, Schoepf UJ, Silverman JR, McWhite CB, Krazinski AW, Bayer RR and Meinel FG: Incremental value of pharmacological stress cardiac dual-energy ct over coronary ct angiography alone for the assessment of coronary artery disease in a high-risk population. AJR Am J Roentgenol 203(1): W70-77, 2014.

6 Meinel FG, De Cecco CN, Schoepf UJ, Nance JW Jr., Silverman JR, Flowers BA and Henzler T: First-arterial-pass dual-energy ct for assessment of myocardial blood supply: Do we need rest, stress, and delayed acquisition? Comparison with spect. Radiology 270(3): 708-716, 2014.

7 Leipsic J, Abbara S, Achenbach S, Cury R, Earls JP, Mancini GJ, Nieman K, Pontone G and Raff GL: Scct guidelines for the interpretation and reporting of coronary ct angiography: A report of the society of cardiovascular computed tomography guidelines committee. J Cardiovasc Comput Tomogr 8(5): 342358, 2014.
8 Douglas PS, Pontone G, Hlatky MA, Patel MR, Norgaard BL, Byrne RA, Curzen N, Purcell I, Gutberlet M, Rioufol G, Hink U, Schuchlenz HW, Feuchtner G, Gilard M, Andreini D, Jensen JM, Hadamitzky M, Chiswell K, Cyr D, Wilk A, Wang F, Rogers $\mathrm{C}$ and De Bruyne B: Clinical outcomes of fractional flow reserve by computed tomographic angiography-guided diagnostic strategies vs. Usual care in patients with suspected coronary artery disease: The prospective longitudinal trial of $\mathrm{ffr}(\mathrm{ct})$ : Outcome and resource impacts study. Eur Heart J 36(47): 33593367, 2015.

9 Cannao PM, Schoepf UJ, Muscogiuri G, Wichmann JL, Fuller SR, Secchi F, Varga-Szemes A and De Cecco CN: Technical prerequisites and imaging protocols for dynamic and dual energy myocardial perfusion imaging. Eur J Radiol 84(12): 2401-2410, 2015.

10 Osawa K, Miyoshi T, Miki T, Koyama Y, Sato S, Kanazawa S and Ito H: Diagnostic performance of first-pass myocardial perfusion imaging without stress with computed tomography (ct) compared with coronary ct angiography alone, with fractional flow reserve as the reference standard. PLoS One 11(2): e0149170, 2016.

11 Meyer M, Haubenreisser H, Schoepf UJ, Vliegenthart R, Leidecker C, Allmendinger T, Lehmann R, Sudarski S, Borggrefe M, Schoenberg SO and Henzler T: Closing in on the k edge: Coronary ct angiography at 100,80 , and $70 \mathrm{kv}$-initial comparison of a second-versus a third-generation dual-source ct system. Radiology 273(2): 373-382, 2014.

12 Coenen A, Rossi A, Lubbers MM, Kurata A, Kono AK, Chelu RG, Segreto S, Dijkshoorn ML, Wragg A, van Geuns RM, Pugliese $\mathrm{F}$ and Nieman K: Integrating ct myocardial perfusion and ct-ffr in the work-up of coronary artery disease. JACC Cardiovasc Imaging 10(7): 760-770, 2017.

13 Genders TS, Steyerberg EW, Alkadhi H, Leschka S, Desbiolles L, Nieman K, Galema TW, Meijboom WB, Mollet NR, de Feyter PJ, Cademartiri F, Maffei E, Dewey M, Zimmermann E, Laule M, Pugliese F, Barbagallo R, Sinitsyn V, Bogaert J, Goetschalckx K, Schoepf UJ, Rowe GW, Schuijf JD, Bax JJ, de Graaf FR, Knuuti J, Kajander S, van Mieghem CA, Meijs MF, Cramer MJ, Gopalan D, Feuchtner G, Friedrich G, Krestin GP, Hunink MG and Consortium CAD: A clinical prediction rule for the diagnosis of coronary artery disease: Validation, updating, and extension. Eur Heart J 32(11): 1316-1330, 2011.
Received August 4, 2017

Revised August 21, 2017 Accepted August 22, 2017 